\title{
Darboux transformations, finite reduction groups and related Yang-Baxter maps
}

\author{
S. Konstantinou-Rizos and A. V. Mikhailov \\ Department of Applied Mathematics, University of Leeds, Leeds \\ mmskr@leeds.ac.uk, A.V.Mikhailov@leeds.ac.uk
}

November 10, 2018

\begin{abstract}
In this paper we construct Yang-Baxter (YB) maps using Darboux matrices which are invariant under the action of finite reduction groups. We present 6-dimensional YB maps corresponding to Darboux transformations for the Nonlinear Schrödinger (NLS) equation and the derivative Nonlinear Schrödinger (DNLS) equation. These YB maps can be restricted to 4-dimensional YB maps on invariant leaves. The former are completely integrable and they also have applications to a recent theory of maps preserving functions with symmetries 14. We give a 6- dimensional YB-map corresponding to the Darboux transformation for a deformation of the DNLS equation. We also consider vector generalisations of the YB maps corresponding to the NLS and DNLS equation.
\end{abstract}

\section{Introduction}

The Yang-Baxter equation has a fundamental role in the theory of quantum and classical integrable systems. In particular, Yang-Baxter maps, namely the set theoretical solutions [12] of the Yang-Baxter equation have been of great interest for several researchers in the area of Mathematical Physics. They are related to several concepts of integrability as, for instance, the multidimensionally consistent equations [3, 4, 7, 27, 28, 29]. Especially, for those Yang-Baxter maps which admit Lax representation [34, there are corresponding hierarchies of commuting transfer maps which preserve the spectrum of their monodromy matrix [36, 37]. Therefore, the construction of Yang-Baxter maps is important.

There are several methods of construction of Yang-Baxter maps, coming from several fields of mathematics as the quantum group theory, representation theory, Lie group theory etc. Here, we construct Yang-Baxter maps using Darboux transformations for Lax operators of integrable partial differential equations. These Yang-Baxter maps can be reduced to completely itnegrable maps on invariant leaves.

The Yang-Baxter equation

$$
Y^{12} \circ Y^{13} \circ Y^{23}=Y^{23} \circ Y^{13} \circ Y^{12},
$$

originates in the works of Yang [38] and Baxter [6]. Here $Y^{i j}$ denotes the action of a linear operator $Y: U \otimes U \rightarrow U \otimes U$ on the $i j$ factor of the triple tensor product $U \otimes U \otimes U$, where 
$U$ is a vector space. In this form, equation (1) is known in the literature as the quantum $Y B$ equation.

Drinfel'd in 1992 [12] proposed to replace $U$ by an arbitrary set $A$ and, therefore, the tensor product $U \otimes U$ by the Cartesian product $A \times A$. In our paper $A$ is an algebraic variety in $K^{N}$, where $K$ is any field of zero characteristic, such us $\mathbb{C}$ or $\mathbb{Q}$.

In [36] Veselov proposed the term Yang Baxter map for the set-theoretical solutions of the quantum YB equation. Specifically, we consider the map $Y: A \times A \rightarrow A \times A$,

$$
Y:(x, y) \mapsto(u(x, y), v(x, y)) .
$$

Furthermore, we define the functions $Y^{i, j}: A \times A \times A \rightarrow A \times A \times A$ for $i, j=1,2,3, i \neq j$, which appear in equation (1), by the following relations

$$
\begin{aligned}
& Y^{12}(x, y, z)=(u(x, y), v(x, y), z) \\
& Y^{13}(x, y, z)=(u(x, z), y, v(x, z)), \\
& Y^{23}(x, y, z)=(x, u(y, z), v(y, z))
\end{aligned}
$$

where $x, y, z \in A$. The variety $A$, in general, can be of any dimension. Thus, elements $x \in A$ are points in $K^{N}$. The map $Y^{j i}, i<j$, is defined as $Y^{i j}$ where we swap $u(k, l) \leftrightarrow v(l, k)$, $k, l=x, y, z$. For example, $Y^{21}(x, y, z)=(v(y, x), u(y, x), z)$.

The map (2) is a YB map, if it satisfies the YB equation (11). Moreover, it is called reversible if the composition of $Y^{i j}$ and $Y^{j i}$ is the identity map,

$$
Y^{i j} \circ Y^{j i}=I d \text {. }
$$

We use the term parametric $Y B$ map when $u$ and $v$ are attached with parameters $a, b \in K^{n}$, namely $u=u(x, y ; a, b)$ and $v=v(x, y ; a, b)$, meaning that the following map

$$
Y_{a, b}:(x, y ; a, b) \mapsto(u(x, y ; a, b), v(x, y ; a, b)),
$$

satisfies the parametric $Y B$ equation

$$
Y_{a, b}^{12} \circ Y_{a, c}^{13} \circ Y_{b, c}^{23}=Y_{b, c}^{23} \circ Y_{a, c}^{13} \circ Y_{a, b}^{12} .
$$

Following Suris and Veselov in 34, we call a Lax matrix for a parametric YB map, a matrix $L=L(x ; c ; \lambda)$ depending on a variable $x$, a parameter $c$ and a spectral parameter $\lambda$, such that the Lax-equation

$$
L(u ; a, \lambda) L(v ; b, \lambda)=L(y ; b, \lambda) L(x ; a, \lambda), \quad \text { for any } \lambda \in K,
$$

is satisfied due to the YB map. The above is also called a refactorisation problem.

It is obvious that the Lax-equation (9) does not always have a unique solution, which motivated Kouloukas and Papageorgiou in [20] to propose the term strong Lax matrix for a YB map. This is when the Lax-equation is equivalent to a YB map

$$
(u, v)=Y_{a, b}(x, y)
$$


Actually, the uniqueness of refactorisation (9) is a sufficient condition for the solutions of the Lax-equation to define a reversible YB map [18, 20, 37] of the form (10). In the opposite case, one may need to check if the obtained map satisfies the YB equation.

One of the most famous parametric YB maps is Adler's map [1]

$$
\left(x_{1}, x_{2}\right) \longrightarrow(u, v)=\left(x_{2}-\frac{a-b}{x_{1}+x_{2}}, x_{1}+\frac{a-b}{x_{1}+x_{2}}\right),
$$

which occurs from the 3-D consistent discrete potential KDV equation [26, 30. In terms of Lax matrices, Adler's map (11) is obtained from the following strong Lax matrix [34, 37]

$$
L(x ; a, \lambda)=\left(\begin{array}{cc}
x & 1 \\
x^{2}+a-\lambda & x
\end{array}\right) .
$$

In [31, 32] a variety of YB maps is constructed using the symmetries of multi-field equations on quad graphs.

It follows from the structure of the Lax-equation (9) that we can extract invariants of the YB map, which we denote as $I_{i}(x, y)$. The invariants are useful if one is interested in the dynamics of such maps. In terms of dynamics, the most interesting maps are those which are not involutive. Although, involutive maps have also useful applications [15]. In all the cases presented in the next sections, our YB maps are not involutive.

In this paper we are interested in the integrability of the YB maps as finite discrete maps. The transfer dynamics of YB maps is discussed in [37].

Now, following [13, 35] we define integrability for YB maps.

Definition 1.1. A $2 N$ - dimensional Yang-Baxter map,

$$
Y:\left(x_{1}, \ldots, x_{2 N}\right) \mapsto\left(u_{1}, \ldots, u_{2 N}\right), \quad u_{i}=u_{i}\left(x_{1}, \ldots, x_{2 N}\right), \quad i=1, \ldots, 2 N,
$$

is said to be completely integrable or Liouville integrable if

1. there is a Poisson matrix $[J]_{i j}=\left\{x_{i}, x_{j}\right\}$, of rank $2 r$, which is invariant under $Y$, namely the matrix $[\tilde{J}]_{i j}=\left\{u_{i}, u_{j}\right\}$ has the same functional form with $J$,

2. map $Y$ has $r$-functionally independent invariants, which are in involution with respect to the corresponding Poisson bracket, i.e. $\left\{I_{i}, I_{j}\right\}=0, i, j=1, \ldots, r, i \neq j$,

3. there are $k=2 N-2 r$ in the number Casimir functions, $C_{i}, i=1, \ldots, k$, which are invariant under $Y$, namely $C_{i} \circ Y=C_{i}$.

\section{Organisation of the paper}

In the next section we briefly give some introduction to the notion of the reduction group [24, automorphic Lie algebras [8, 9, 21, 22] and Darboux trasformations to make this text self-contained. We state three cases of reduction groups which are representative for all the finite reduction groups with degenerate orbits 21, 22. 
In Section 4 we use Darboux transformations presented in [17] to derive YB maps. In particular, we consider Darboux matrices for the NLS equation, the DNLS equation and for a deformation of the DNLS equation. For these Darboux matrices the refactorisation is not unique. Therefore, for the corresponding 6-dimensional YB maps which are derived from the refactorisation problem, in principle, one needs to check the YB property separately. Yet, the entries of these Darboux matrices obey certain differential equations which possess first integrals. There is a natural restriction of the Darboux map on the affine variety corresponding to a level set of these first integrals. These restrictions make the refactorisation unique and this guarantees that the induced 4- dimensional YB maps satisfy the YB equation and they are reversible [37. We show that these YB maps have Poisson structure. However, the first integrals are not always very useful for the reduction because, in general, they are polynomial equations. In fact, in the case of the DNLS equation the corresponding 6-dimensional YB map cannot be reduced to a 4-dimensional one on the invariant leaves explicitly. However, after a change in the variables we obtain a reducible map. In the case of the deformation of the DNLS equation, we can only derive the 6-dimensional YB map and its implicit restriction on 4-dimensional invariant leaves.

In Section 5 we consider the vector generalisations of the Adler-Yamilov YB map and the YB map corresponding to the DNLS equation.

\section{Automorphic Lie algebras and Darboux Transformations and reduction groups with degenerate orbits}

The reduction group was first introduced in 24]. It is a discrete group of automorphisms of a Lax operator and its elements are silmutaneous gauge transformations and fractional-linear transformations of the spectral parameter.

Automorphic Lie algebras were introduced in [21, 22] and studied in [8, 9, 21, 22]. These

algebras constitute a subclass of infinite dimensional Lie algebras and their name is due to their construction which is very similar to the one for automorphic functions.

Darboux transformations and their relations to the theory of integrable systems have been extensively studied [23, 33. Such transformations can be derived from Lax pairs as, for instance, in [33, or in a more systematic algebraic manner in [11, 17].

We are interested in Darboux transformations corresponding to Lax operators of the following form

$$
\mathfrak{L}=\mathfrak{L}(\mathbf{p}(x) ; \lambda)=D_{x}+U(\mathbf{p}(x) ; \lambda),
$$

where $U$ belongs to an automorphic Lie algebra.

In the rest of the text we use " $\mathfrak{L}$ " for Lax operators and " $L$ " for Lax matrices of the refactorisation problem (9).

By Darboux transformations we understand maps

$$
\mathfrak{L}(\mathbf{p}(x) ; \lambda) \rightarrow \widetilde{\mathfrak{L}}:=\mathfrak{L}(\widetilde{\mathbf{p}}(x) ; \lambda)=M \mathfrak{L} M^{-1},
$$

$M$ is a matrix called the Darboux matrix. They map fundamental solutions, $\Psi$, of the equation $\mathfrak{L} \Psi=0$ to other fundamental solutions, $\widetilde{\Psi}=M \Psi$, of the equation $\widetilde{\mathfrak{L}} \widetilde{\Psi}=0$. 
The structure of Lax operators has a natural Lie algebraic interpretation in terms of KacMoody algebras and automorphic Lie algebras [8, 9, 21, 22]. While a Kac-Moody algebra is associated with an automorphism of finite order, automorphic Lie algebras correspond to a finite group of automorphisms, namely the reduction group [24].

In [8, 9] it was proved that in the case of $2 \times 2$ matrices, which we study in this paper, the essentially different reduction groups are the trivial group (with no reduction), the cyclic group $\mathbb{Z}_{2}$ (leading to the Kac-Moody algebra $A_{1}^{1}$ ) and the Klein group $\mathbb{Z}_{2} \times \mathbb{Z}_{2}$, 22, 25].

We shall present 4 and 6 -dimensional YB maps for all the following cases. The trivial group associated with the NLS equation [39]

$$
p_{t}=p_{x x}+4 p^{2} q, \quad q_{t}=-q_{x x}-4 p q^{2} .
$$

The $\mathbb{Z}_{2}$ group associated to the DNLS equation equation [16]

$$
p_{t}=p_{x x}+4\left(p^{2} q\right)_{x}, \quad q_{t}=-q_{x x}+4\left(p q^{2}\right)_{x} .
$$

and the $\mathbb{Z}_{2} \times \mathbb{Z}_{2}$ group associated to the deformation of the DNLS equation [25]

$$
p_{t}=p_{x x}+8\left(p^{2} q\right)_{x}-4 q_{x}, \quad q_{t}=-q_{x x}+8\left(p q^{2}\right)_{x}-4 p_{x} .
$$

The above mentioned groups are representative of all the finite reduction groups with degenerate orbits, namely orbits corresponding to the fixed points of the fractional linear transformations of the spectral parameter.

\section{Derivation of YB maps}

In 17] we used Darboux transformations to construct integrable systems of discrete equations, which have the multidimensional consistency property [3, 4, 7, 27, 28, 29]. The compatibility condition of Darboux transformations around the square is exactly the same with the Lax equation (9). Therefore, in this paper, we use Darboux transformations to construct YB maps.

We start with the well known example of the Darboux transformation for the nonlinear Schrödinger equation and construct its associated YB map.

\subsection{The Nonlinear Schrödinger equation}

In the case of NLS equation, the Lax operator is given by

$$
\mathfrak{L}(p, q ; \lambda)=D_{x}+\lambda U_{1}+U_{0}, \quad \text { where } \quad U_{1}=\sigma_{3}=\operatorname{diag}(1,-1), \quad U_{0}=\left(\begin{array}{cc}
0 & 2 p \\
2 q & 0
\end{array}\right) .
$$

The Darboux Transformation, $M$, of $\mathfrak{L}$ is given by [17, 33]

$$
M=\lambda\left(\begin{array}{ll}
1 & 0 \\
0 & 0
\end{array}\right)+\left(\begin{array}{ll}
f & p \\
\widetilde{q} & 1
\end{array}\right)
$$


The entries of (19), according to definition (14), must satisfy the following system of equations

$$
\partial_{x} f=2(p q-\widetilde{p} \widetilde{q}), \quad \partial_{x} p=2(p f-\widetilde{p}), \quad \partial_{x} \widetilde{q}=2(q-\widetilde{q} f),
$$

which admits the following first integral

$$
\partial_{x}(f-p \widetilde{q})=0
$$

This integral implies that $\partial_{x} \operatorname{det} M=0$.

In correspondence with (19), we define the matrix

$$
M(\mathbf{x}, X ; \lambda)=\lambda\left(\begin{array}{cc}
1 & 0 \\
0 & 0
\end{array}\right)+\left(\begin{array}{cc}
X & x_{1} \\
x_{2} & 1
\end{array}\right)
$$

and substitute it into the Lax equation (9)

$$
M(\mathbf{u}, U ; \lambda) M(\mathbf{v}, V ; \lambda)=M(\mathbf{y}, Y ; \lambda) M(\mathbf{x}, X ; \lambda),
$$

to derive the following system of equations

$$
\begin{gathered}
v_{1}=x_{1}, u_{2}=y_{2}, U+V=X+Y, u_{2} v_{1}=x_{1} y_{2}, \\
u_{1}+U v_{1}=y_{1}+x_{1} Y, u_{1} v_{2}+U V=x_{2} y_{1}+X Y, v_{2}+u_{2} V=x_{2}+X y_{2} .
\end{gathered}
$$

The corresponding algebraic variety is a union of two six-dimensional components. The first one is obvious from the refactorisation problem (22), and it corresponds to the permutation map

$$
\mathbf{x} \mapsto \mathbf{u}=\mathbf{y}, \quad \mathbf{y} \mapsto \mathbf{v}=\mathbf{x}, \quad X \mapsto U=Y, \quad Y \mapsto V=X,
$$

which is a trivial YB map. The second one can be represented as a rational 6-dimensional non-involutive map of $K^{3} \times K^{3} \rightarrow K^{3} \times K^{3}$

$$
\begin{array}{ll}
x_{1} \mapsto u_{1}=\frac{y_{1}+x_{1}^{2} x_{2}-x_{1} X+x_{1} Y}{1+x_{1} y_{2}}, & y_{1} \mapsto v_{1}=x_{1}, \\
x_{2} \mapsto u_{2}=y_{2}, & y_{2} \mapsto v_{2}=\frac{x_{2}+y_{1} y_{2}^{2}+y_{2} X-y_{2} Y}{1+x_{1} y_{2}}, \\
X \mapsto U=\frac{y_{1} y_{2}-x_{1} x_{2}+X+x_{1} y_{2} Y}{1+x_{1} y_{2}}, & Y \mapsto V=\frac{x_{1} x_{2}-y_{1} y_{2}+x_{1} y_{2} X+Y}{1+x_{1} y_{2}},
\end{array}
$$

which, one can easily check that, statisfies the YB equation.

It follows from (22) that the trace of $M(\mathbf{y}, Y ; \lambda) M(\mathbf{x}, X ; \lambda)$, is a polynomial in $\lambda$ whose coefficients are

$$
\operatorname{Tr}(M(\mathbf{y} ; b, \lambda) M(\mathbf{x} ; a, \lambda))=\lambda^{2}+\lambda I_{1}(\mathbf{x}, \mathbf{y}, X, Y)+I_{2}(\mathbf{x}, \mathbf{y}, X, Y),
$$

where

$$
I_{1}(\mathbf{x}, \mathbf{y}, X, Y)=X+Y \quad \text { and } \quad I_{2}(\mathbf{x}, \mathbf{y}, X, Y)=x_{2} y_{1}+x_{1} y_{2}+X Y,
$$

and those are invariants for the YB map (23).

In the following section we show that the YB map (23) can be reduced to a 4 - dimensional YB map which has Poisson structure. 


\subsubsection{Restriction on invariant leaves: The Adler-Yamilov map}

We now take into account the first integral (20) of the Darboux matrix (19). This first integral requires that the matrix entries $f, q$ and $\widetilde{q}$ are related as

$$
f-p \widetilde{q}=\alpha=\text { constant }
$$

Therefore, the entries of matrix (21) must obey the following equation

$$
X-x_{1} x_{2}=a,
$$

respectively.

The matrix (21) now takes the form

$$
M(\mathbf{x} ; a, \lambda)=\lambda\left(\begin{array}{ll}
1 & 0 \\
0 & 0
\end{array}\right)+\left(\begin{array}{cc}
a+x_{1} x_{2} & x_{1} \\
x_{2} & 1
\end{array}\right) .
$$

In this case, the Lax equation (9),

$$
M(\mathbf{u} ; a, \lambda) M(\mathbf{v} ; b, \lambda)=M(\mathbf{y} ; b, \lambda) M(\mathbf{x} ; a, \lambda),
$$

has a unique solution $\mathbf{u}=\mathbf{u}(\mathbf{x}, \mathbf{y}), \mathbf{v}=\mathbf{v}(\mathbf{x}, \mathbf{y})$ which defines a map $\mathbf{x} \rightarrow \mathbf{u}(\mathbf{x}, \mathbf{y}), \mathbf{y} \rightarrow$ $\mathbf{v}(\mathbf{x}, \mathbf{y})$, given by

$$
(\mathbf{x}, \mathbf{y}) \stackrel{Y_{a, b}}{\longrightarrow}\left(y_{1}-\frac{a-b}{1+x_{1} y_{2}} x_{1}, y_{2}, x_{1}, x_{2}+\frac{a-b}{1+x_{1} y_{2}} y_{2}\right) .
$$

Therefore, the above map is a reversible parametric YB map with strong Lax matrix (26). Moreover, it is not involutive.

The map (28) first appeared in the work of Adler Yamilov [5]. Its interpretation as a YB map was given in [19, 31.

From the trace of $M(\mathbf{y} ; b, \lambda) M(\mathbf{x} ; a, \lambda)$ we obtain the following invariants for the map (28)

$$
\begin{aligned}
& I_{1}(\mathbf{x}, \mathbf{y})=x_{1} x_{2}+y_{1} y_{2}+a+b \\
& I_{2}(\mathbf{x}, \mathbf{y})=\left(a+x_{1} x_{2}\right)\left(b+y_{1} y_{2}\right)+x_{1} y_{2}+x_{2} y_{1}+1 .
\end{aligned}
$$

The constant terms in $I_{1}, I_{2}$ can be omitted. It is easy to check that $I_{1}, I_{2}$ are in involution with respect to invariant Poisson brackets defined as

$$
\left\{x_{1}, x_{2}\right\}=\left\{y_{1}, y_{2}\right\}=1, \quad \text { and all the rest } \quad\left\{x_{i}, y_{j}\right\}=0,
$$

and the corresponding Poisson matrix is invariant under the YB map (28). Therefore the map (28) is completely integrable.

The Adler-Yamilov map is a restriction of the YB map (23) on the invariant leaves

$$
A_{a}=\left\{\left(x_{1}, x_{2}, X\right) \in \mathbb{R}^{3} ; X=a+x_{1} x_{2}\right\}, \quad B_{b}=\left\{\left(y_{1}, y_{2}, Y\right) \in \mathbb{R}^{3} ; Y=b+y_{1} y_{2}\right\} .
$$




\subsection{Derivative NLS equation: $\mathbb{Z}_{2}$ reduction}

The Lax operator for the DNLS equation [10, 16] is given by

$$
\mathfrak{L}(p, q ; \lambda)=D_{x}+\lambda^{2} U_{2}+\lambda U_{1}, \quad \text { where } \quad U_{2}=\sigma_{3}, \quad U_{1}=\left(\begin{array}{cc}
0 & 2 p \\
2 q & 0
\end{array}\right),
$$

and $\sigma_{3}$ is a Pauli matrix. Operator $\mathfrak{L}$ is invariant with respect to the following involution

$$
\mathfrak{L}(\lambda)=\sigma_{3} \mathfrak{L}(-\lambda) \sigma_{3},
$$

where $\mathfrak{L}(\lambda) \equiv \mathfrak{L}(p, q ; \lambda)$. Involution (34) generates the so-called reduction group [24, 22, and it is isomorphic to $\mathbb{Z}_{2}$.

The Darboux matrix in this case is given by [17.

$$
M:=\lambda^{2}\left(\begin{array}{cc}
f & 0 \\
0 & 0
\end{array}\right)+\lambda\left(\begin{array}{cc}
0 & f p \\
f \widetilde{q} & 0
\end{array}\right)+\left(\begin{array}{cc}
c & 0 \\
0 & 1
\end{array}\right),
$$

whose entries $p, \widetilde{q}$ and $f$ obey the following system of equations

$$
\begin{aligned}
\partial_{x} p & =2 p(\widetilde{p} \widetilde{q}-p q)-\frac{2}{f}(\widetilde{p}-c p), \\
\partial_{x} \widetilde{q} & =2 \widetilde{q}(\widetilde{p} \widetilde{q}-p q)-\frac{2}{f}(c \widetilde{q}-q), \\
\partial_{x} f & =2 f(p q-\widetilde{p} \widetilde{q}) .
\end{aligned}
$$

System (36)-(38) has a first integral which obliges the determinant of matrix (35) to be $x$-independent, and it is given by

$$
\partial_{x}\left(f^{2} p \widetilde{q}-f\right)=0
$$

Using the entries of (35) as variables, namely $(p, \widetilde{q}, f ; c) \rightarrow\left(x_{1}, x_{2}, X ; 1\right)$, we define the matrix

$$
M(\mathbf{x}, X ; \lambda)=\lambda^{2}\left(\begin{array}{cc}
X & 0 \\
0 & 0
\end{array}\right)+\lambda\left(\begin{array}{cc}
0 & x_{1} X \\
x_{2} X & 0
\end{array}\right)+\left(\begin{array}{cc}
1 & 0 \\
0 & 1
\end{array}\right) .
$$

The Lax equation implies the following equations

$$
\begin{gathered}
u_{1} U+v_{1} V=x_{1} X+y_{1} Y, \quad u_{2} U+v_{2} V=x_{2} X+y_{2} Y, \\
U V=X Y, \quad v_{1} U V=x_{1} X Y, \quad u_{2} U V=y_{2} X Y, \quad u_{2} v_{1} U V=x_{1} y_{2} X Y, \\
U+V+u_{1} v_{2} U V=X+Y+x_{2} y_{1} X Y .
\end{gathered}
$$

As in the case of nonlinear Schrödinger equation, the algebraic variety consists of two components. The first 6 -dimensional component corresponds to the permutation map

$$
\mathbf{x} \mapsto \mathbf{u}=\mathbf{y}, \quad \mathbf{y} \mapsto \mathbf{v}=\mathbf{x}, \quad X \mapsto U=Y, \quad Y \mapsto V=X,
$$

and the second corresponds to the following 6-dimensional YB map

$$
\begin{aligned}
x_{1} & \mapsto u_{1}=f_{1}(\mathbf{x}, \mathbf{y}, X, Y), & y_{1} & \mapsto v_{1}=f_{2}(\pi \mathbf{y}, \pi \mathbf{x}, Y, X), \\
x_{2} & \mapsto u_{2}=f_{2}(\mathbf{x}, \mathbf{y}, X, Y), & y_{2} & \mapsto v_{2}=f_{1}(\pi \mathbf{y}, \pi \mathbf{x}, Y, X), \\
X & \mapsto U=f_{3}(\mathbf{x}, \mathbf{y}, X, Y), & Y & \mapsto V=f_{3}(\pi \mathbf{y}, \pi \mathbf{x}, Y, X),
\end{aligned}
$$


where $\pi$ is the permutation function, $\pi\left(x_{1}, x_{2}\right)=\left(x_{2}, x_{1}\right), \pi^{2}=1$ and $f_{1}, f_{2}$ and $f_{3}$ are given by

$$
\begin{aligned}
f_{1}(\mathbf{x}, \mathbf{y}, X, Y) & =\frac{-1}{f_{3}(\mathbf{x}, \mathbf{y})} \frac{x_{1} X+\left(y_{1}-x_{1}\right) Y-x_{1} x_{2} y_{1} X Y-x_{1}^{2} x_{2} X^{2}}{x_{1} x_{2} X+x_{1} y_{2} Y-1} \\
f_{2}(\mathbf{x}, \mathbf{y}, X, Y) & =y_{2} \\
f_{3}(\mathbf{x}, \mathbf{y}, X, Y) & =\frac{x_{1} x_{2} X+x_{1} y_{2} Y-1}{x_{1} y_{2} X+y_{1} y_{2} Y-1} X .
\end{aligned}
$$

One can verify that the above map is a non-involutive YB map. The invariants of this map are given by

$$
I_{1}(\mathbf{x} \cdot \pi \mathbf{y}, X, Y)=X Y \quad \text { and } \quad I_{2}(\mathbf{x}, \mathbf{y}, X, Y)=(\mathbf{x} \cdot \pi \mathbf{y}) X Y+X+Y .
$$

However, this map cannot be easily reduced on invariant leaves as in the case of NLS equation. This is due to the fact that the first integral of system (36)-(38) is

$$
f-f^{2} p \widetilde{q}=k,
$$

and therefore the relation between the entries $x_{1}, x_{2}$ and $X$ of (40) reads

$$
X-X^{2} x_{1} x_{2}=k .
$$

The solution for $X$ of the above equation can only be expressed in terms of square roots of $x_{1}$ and $x_{2}$. Thus, in this case is difficult to solve the Lax equation, and if we do so the resulting map will not be presentable because of its length.

One option is to present the YB map implicitly. Specifically, the corresponding 4-dimensional map is given by

$$
\begin{array}{ll}
x_{1} \mapsto u_{1}=f_{1}(\mathbf{x}, \mathbf{y}, X, Y), & y_{1} \mapsto v_{1}=f_{2}(\pi \mathbf{y}, \pi \mathbf{x}, Y, X), \\
x_{2} \mapsto u_{2}=f_{2}(\mathbf{x}, \mathbf{y}, X, Y), & y_{2} \mapsto v_{2}=f_{1}(\pi \mathbf{y}, \pi \mathbf{x}, Y, X),
\end{array}
$$

where $f_{i}, i=1,2$, are given by (44)-(45) where $X$ and $Y$ are given by

$$
X-X^{2} x_{1} x_{2}=a, \quad Y-Y^{2} y_{1} y_{2}=b .
$$

Nevertheless, we can choose another parametrisation of matrix (35), in order to make the obtained YB map explicitly reducible on the invariant leaves.

\subsection{1 $\mathbb{Z}_{2}$ reduction: A reducible 6-dimensional YB map}

Now, lets go back to the Darboux matrix (35) and replace $(f p, f \widetilde{q}, f ; c) \rightarrow\left(x_{1}, x_{2}, X ; 1\right)$, namely

$$
M(\mathbf{x}, X ; \lambda)=\lambda^{2}\left(\begin{array}{cc}
X & 0 \\
0 & 0
\end{array}\right)+\lambda\left(\begin{array}{cc}
0 & x_{1} \\
x_{2} & 0
\end{array}\right)+\left(\begin{array}{cc}
1 & 0 \\
0 & 1
\end{array}\right)
$$

From the Lax equation we obtain the following equations

$$
\begin{array}{r}
u_{2} v_{1}=x_{1} y_{2}, \quad u_{2} V=X y_{2}, \quad U v_{1}=x_{1} Y, \quad U V=X Y \\
u_{1}+v_{1}=x_{1}+y_{1}, \quad U+u_{1} v_{2}+V=X+x_{2} y_{1}+Y, \quad u_{2}+v_{2}=x_{2}+y_{2} .
\end{array}
$$


Now, the first 6-dimensional component of the algebraic variety corresponds to the trivial map (42) and the second component corresponds to a map of the form (43), with $f_{1}, f_{2}$ and $f_{3}$ now given by

$$
\begin{aligned}
f_{1}(\mathbf{x}, \mathbf{y}, X, Y) & =\frac{\left(x_{1}+y_{1}\right) X-x_{1} Y-x_{1} x_{2}\left(x_{1}+y_{1}\right)}{X-x_{1}\left(x_{2}+y_{2}\right)} \\
f_{2}(\mathbf{x}, \mathbf{y}, X, Y) & =\frac{X-x_{1}\left(x_{2}+y_{2}\right)}{Y-y_{2}\left(x_{1}+y_{1}\right)} y_{2} \\
f_{3}(\mathbf{x}, \mathbf{y}, X, Y) & =\frac{X-x_{1}\left(x_{2}+y_{2}\right)}{Y-y_{2}\left(x_{1}+y_{1}\right)} Y .
\end{aligned}
$$

This map has the following invariants

$$
\begin{array}{r}
I_{1}(\mathbf{x}, \mathbf{y}, X, Y)=X Y, \quad I_{2}(\mathbf{x}, \mathbf{y}, X, Y)=\mathbf{x} \cdot \pi \mathbf{y}+X+Y, \\
I_{3}(\mathbf{x}, \mathbf{y}, X, Y)=x_{1}+y_{1}, \quad I_{4}(\mathbf{x}, \mathbf{y}, X, Y)=x_{2}+y_{2} .
\end{array}
$$

\subsubsection{Restriction on invariant leaves}

Now, taking into account the first integral

$$
f-(f p)(f \widetilde{q})=k=\text { constant }
$$

the entries of matrix (52) must satisfy the following equation

$$
X-x_{1} x_{2}=k,
$$

and thus matrix (52) takes the following form

$$
M(\mathbf{x} ; k ; \lambda)=\lambda^{2}\left(\begin{array}{cc}
k+x_{1} x_{2} & 0 \\
0 & 0
\end{array}\right)+\lambda\left(\begin{array}{cc}
0 & x_{1} \\
x_{2} & 0
\end{array}\right)+\left(\begin{array}{cc}
1 & 0 \\
0 & 1
\end{array}\right) .
$$

The Lax-equation for matrix (60) is equivalent to the following

$$
(\mathbf{x}, \mathbf{y}) \stackrel{Y_{a, b}}{\longrightarrow}\left(y_{1}+\frac{a-b}{a-x_{1} y_{2}} x_{1}, \frac{a-x_{1} y_{2}}{b-x_{1} y_{2}} y_{2}, \frac{b-x_{1} y_{2}}{a-x_{1} y_{2}} x_{1}, x_{2}+\frac{b-a}{b-x_{1} y_{2}} y_{2}\right)
$$

and therefore the above map is a reversible parametric YB map. Moreover, it is not involutive.

The invariants of map (61) are given by

$$
I_{1}(\mathbf{x}, \mathbf{y})=\left(a+x_{1} x_{2}\right)\left(b+y_{1} y_{2}\right), \quad I_{2}(\mathbf{x}, \mathbf{y})=\left(x_{1}+y_{1}\right)\left(x_{2}+y_{2}\right)+a+b .
$$

The constant terms in $I_{1}$ and $I_{2}$ can be omitted. These are the invariants we retrieve from the trace of $M(\mathbf{y} ; b, \lambda) M(\mathbf{x} ; a, \lambda)$. However, the quantities $x_{1}+y_{1}$ and $x_{2}+y_{2}$ in $I_{2}$ are invariants themselves. The Poisson bracket in this case is given by

$$
\left\{x_{1}, x_{2}\right\}=\left\{y_{1}, y_{2}\right\}=\left\{x_{2}, y_{1}\right\}=\left\{y_{2}, x_{1}\right\}=1, \quad \text { and all the rest } \quad\left\{x_{i}, y_{j}\right\}=0
$$


The rank of the Poisson matrix is $2, I_{1}$ is one invariant and $I_{2}=C_{1} C_{2}+a+b$, where $C_{1}=x_{1}+y_{1}$ and $C_{2}=x_{2}+y_{2}$ are Casimir functions. The latter are preserved by (61), namely $C_{i} \circ Y_{a, b}=C_{i}, i=1,2$. Therefore, map (61) is completely integrable.

Map (61) is a restriction of the YB map (53)-(55) on the invariant leaves

$$
A_{a}=\left\{\left(x_{1}, x_{2}, X\right) \in \mathbb{R}^{3} ; X=a+x_{1} x_{2}\right\}, \quad B_{b}=\left\{\left(y_{1}, y_{2}, Y\right) \in \mathbb{R}^{3} ; Y=b+y_{1} y_{2}\right\} .
$$

Moreover, the map (61) can be expressed as a map of two variables on the symplectic leaf

$$
x_{1}+y_{1}=c_{1}, \quad x_{2}+y_{2}=c_{2}
$$

\subsection{A deformation of the DNLS equation: Dihedral Group}

In the case of dihedral reduction group, the Lax operator is given by

$$
\begin{gathered}
\mathfrak{L}(p, q ; \lambda)=D_{x}+\lambda^{2} U_{2}+\lambda U_{1}+\lambda^{-1} U_{-1}-\lambda^{-2} U_{-2}, \quad \text { where } \\
U_{2} \equiv U_{-2}=\sigma_{3}, \quad U_{1}=\left(\begin{array}{cc}
0 & 2 p \\
2 q & 0
\end{array}\right) \quad U_{-1}=\sigma_{1} U_{1} \sigma_{1},
\end{gathered}
$$

and $\sigma_{1}, \sigma_{3}$ are Pauli matrices. Here, the reduction group consists of the following set of transformations acting on the Lax operator (66),

$$
\mathfrak{L}(\lambda)=\sigma_{3} \mathfrak{L}(-\lambda) \sigma_{3} \quad \text { and } \quad \mathfrak{L}(\lambda)=\sigma_{1} \mathfrak{L}\left(\lambda^{-1}\right) \sigma_{1},
$$

and it is isomorphic to $\mathbb{Z}_{2} \times \mathbb{Z}_{2} \cong D_{2},[22$.

In this case, the Darboux matrix is given by [17]

$$
M=f\left(\left(\begin{array}{cc}
\lambda^{2} & 0 \\
0 & \lambda^{-2}
\end{array}\right)+\lambda\left(\begin{array}{cc}
0 & p \\
\widetilde{q} & 0
\end{array}\right)+g\left(\begin{array}{ll}
1 & 0 \\
0 & 1
\end{array}\right)+\frac{1}{\lambda}\left(\begin{array}{ll}
0 & \widetilde{q} \\
p & 0
\end{array}\right)\right)
$$

where its entries obey the following equations

$$
\begin{array}{r}
\partial_{x} p=2((\widetilde{p} \widetilde{q}-p q) p+(p-\widetilde{p}) g+q-\widetilde{q}), \\
\partial_{x} \widetilde{q}=2((\widetilde{p} \widetilde{q}-p q) \widetilde{q}+p-\widetilde{p}+(q-\widetilde{q}) g), \\
\partial_{x} g=2((\widetilde{p} \widetilde{q}-p q) g+(p-\widetilde{p}) p+(q-\widetilde{q}) \widetilde{q}), \\
\left.\partial_{x} f=-2(\widetilde{p} \widetilde{q}-p q)\right) f .
\end{array}
$$

It can be shown that the above system of differential equations admits two first integrals, $\partial_{x} \Phi_{i}=0, i=1,2$, where

$$
\Phi_{1}:=f^{2}(g-p \widetilde{q}) \quad \text { and } \quad \Phi_{2}:=f^{2}\left(g^{2}+1-p^{2}-\widetilde{q}^{2}\right) .
$$

It follows from (73), that the quantities $f$ and $f g$ can be expressed in terms of $p$ and $\widetilde{q}$, as solutions of quadratic equations. Then, the Darboux matrix depends only on two variables and we construct a 4-dimensional parametric YB map. Although, we have omitted this map because of its length.

In the next section we construct a 6 -dimensional map from (68). 


\subsubsection{Dihedral group: A 6-dimensional YB map}

We now consider the matrix $N:=f M$, where $M$ is given by (68), and we change $\left(p, \widetilde{q}, f^{2}\right) \rightarrow$ $\left(x_{1}, x_{2}, X\right)$. Then,

$$
N\left(\mathbf{x}, X ; c_{1}, \lambda\right)=\left(\begin{array}{cc}
\lambda^{2} X+x_{1} x_{2} X+c_{1} & \lambda x_{1} X+\lambda^{-1} x_{2} X \\
\lambda x_{2} X+\lambda^{-1} x_{1} X & \lambda^{-2} X+x_{1} x_{2} X+c_{1}
\end{array}\right),
$$

where we have substituted the product $f^{2} g$ by

$$
f^{2} g=c_{1}+x_{1} x_{2} X
$$

using the first integral, $\Phi_{1}$, in (73).

The Lax equation for the Darboux matrix (74) reads

$$
N(\mathbf{u}, U ; a, \lambda) N(\mathbf{v}, V ; b, \lambda)=N(\mathbf{y}, Y ; b, \lambda) N(\mathbf{x}, X ; a, \lambda),
$$

from where we obtain an algebraic system of equations, omitted because of its length.

The first 6-dimensional component of the corresponding algebraic variety corresponds to the trivial YB map

$$
\mathbf{x} \mapsto \mathbf{u}=\mathbf{y}, \quad \mathbf{y} \mapsto \mathbf{v}=\mathbf{x}, \quad X \mapsto U=\frac{a}{b} Y, \quad Y \mapsto V=\frac{b}{a} X,
$$

and the second component corresponds to the following map

$$
\begin{array}{rlrl}
x_{1} & \mapsto u_{1}=\frac{f(\mathbf{x}, \mathbf{y}, X, Y ; a, b)}{g(\mathbf{x}, \mathbf{y}, X, Y ; a, b)}, & y_{1} \mapsto v_{1}=x_{1} \\
x_{2} \mapsto u_{2}=y_{2}, & y_{2} \mapsto v_{2}=\frac{f(\pi \mathbf{y}, \pi \mathbf{x}, Y, X ; b, a)}{g(\pi \mathbf{y}, \pi \mathbf{x}, Y, X ; b, a)} \\
X \mapsto U=\frac{g(\mathbf{x}, \mathbf{y}, X, Y ; a, b)}{h(\mathbf{x}, \mathbf{y}, X, Y ; a, b)}, & Y \mapsto V=\frac{g(\pi \mathbf{y}, \pi \mathbf{x}, Y, X ; b, a)}{h(\pi \mathbf{y}, \pi \mathbf{x}, Y, X ; b, a)},
\end{array}
$$

where $f, g$ and $h$ are given by

$$
\begin{aligned}
& f(\mathbf{x}, \mathbf{y}, X, Y ; a, b)=a^{2} b^{2} x_{1} X+a^{2} b\left[x_{2}-y_{2}+2 x_{1} x_{2} y_{1}+x_{1}^{2}\left(y_{2}-3 x_{2}\right)\right] X Y+ \\
& a^{2}\left(y_{2}^{2}-1\right)\left[y_{1}\left(1+x_{1}^{2}\right)-x_{1}\left(1+y_{1}^{2}\right)\right] X Y^{2}-a b^{2}\left(x_{1}^{2}-1\right)\left(y_{2}-x_{2}\right) X^{2}- \\
& a b\left(x_{1}^{2}-1\right)\left[x_{2}^{2}\left(3 x_{1}-y_{1}\right)-x_{1}-y_{1}+2 y_{2}\left(y_{1} y_{2}-x_{1} x_{2}\right)\right] X^{2} Y- \\
& a\left(x_{1}^{2}-1\right)\left(y_{2}^{2}-1\right)\left[y_{2}\left(y_{1}^{2}-1\right)+x_{2}\left(y_{1}^{2}-2 x_{1} y_{1}+1\right)\right] X^{2} Y^{2}+ \\
& y_{1}\left(x_{1}^{2}-1\right)^{2}\left(x_{2}^{2}-1\right)\left(y_{2}^{2}-1\right) X^{3} Y^{2}+b\left(x_{1}^{2}-1\right)^{2}\left(x_{2}^{2}-1\right)\left(y_{2}-x_{2}\right) X^{3} Y+ \\
& \quad a^{3} b\left(y_{1}-x_{1}\right) Y, \\
& g(\mathbf{x}, \mathbf{y}, X, Y ; a, b)=a^{2} b^{2} X+2 a^{2} b y_{2}\left(y_{1}-x_{1}\right) X Y+a^{2}\left(y_{2}^{2}-1\right)\left(x_{1}-y_{1}\right)^{2} X Y^{2}+ \\
& \quad 2 a b\left(x_{1}^{2}-1\right)\left(1-x_{2} y_{2}\right) X^{2} Y+2 a x_{2}\left(x_{1}^{2}-1\right)\left(y_{2}^{2}-1\right)\left(x_{1}-y_{1}\right) X^{2} Y^{2}+ \\
& \quad\left(x_{1}^{2}-1\right)^{2}\left(x_{2}^{2}-1\right)\left(y_{2}^{2}-1\right) X^{3} Y^{2}, \\
& h(\mathbf{x}, \mathbf{y}, X, Y ; a, b)=a^{2} b^{2}-2 a b^{2} x_{1}\left(y_{2}-x_{2}\right) X-2 a b\left(x_{1} y_{1}-1\right)\left(y_{2}^{2}-1\right) X Y \\
& \quad b^{2}\left(x_{1}^{2}-1\right)\left(x_{2}-y_{2}\right)^{2} X^{2}-2 b y_{1}\left(x_{2}-y_{2}\right)\left(x_{1}^{2}-1\right)\left(y_{2}^{2}-1\right) X^{2} Y+ \\
& \quad\left(x_{1}^{2}-1\right)\left(y_{1}^{2}-1\right)\left(y_{2}^{2}-1\right)^{2} X^{2} Y^{2} .
\end{aligned}
$$


It can be verified that this is a parametric YB map. From $\operatorname{Tr}(N(\mathbf{x}, X ; \lambda) N(\mathbf{y}, Y ; \lambda))$ we extract the following invariants for the above map

$$
\begin{aligned}
& I_{1}(\mathbf{x}, \mathbf{y}, X, Y ; a, b)=X Y, \\
& I_{2}(\mathbf{x}, \mathbf{y}, X, Y ; a, b)=b X+a Y+\left(x_{1}+y_{1}\right)\left(x_{2}+y_{2}\right) X Y, \\
& I_{3}(\mathbf{x}, \mathbf{y}, X, Y ; a, b)=2 b x_{1} x_{2} X+2 a y_{1} y_{2} Y+2\left(\mathbf{x} \cdot \mathbf{y}+x_{1} x_{2} y_{1} y_{2}\right) X Y+2 a b .
\end{aligned}
$$

\subsubsection{Restriction to invariant leaves: An implicit map}

Using the first integrals $\Phi_{1}$ and $\Phi_{2}$ we can reduce the above 6 -dimensional map to a 4-dimensional YB map implicitly. In particular, from equations $\Phi_{1}=c_{1}$ and $\Phi_{2}=c_{2}$ one can obtain

$$
\left(1-x_{1}^{2}-x_{2}^{2}+x_{1}^{2} x_{2}^{2}\right) X^{2}+\left(2 x_{1} x_{2}-c_{2}\right) X+1=0,
$$

where we have rescaled $c_{1} \rightarrow 1$.

Therefore the 4-dimensional map is given by

$$
\left(u_{1}, u_{2}, v_{1}, v_{2}\right)=\left(\frac{f(\mathbf{x}, \mathbf{y}, X, Y ; a, b)}{g(\mathbf{x}, \mathbf{y}, X, Y ; a, b)}, y_{2}, x_{1}, \frac{f(\pi \mathbf{y}, \pi \mathbf{x}, X, Y ; b, a)}{g(\pi \mathbf{y}, \pi \mathbf{x}, X, Y ; b, a)}\right),
$$

where $f, g$ and $h$ are given by the above relations and $X$ and $Y$ are given by

$$
\begin{array}{r}
\left(1-x_{1}^{2}-x_{2}^{2}+x_{1}^{2} x_{2}^{2}\right) X^{2}+\left(2 x_{1} x_{2}-a\right) X+1=0 \\
\left(1-y_{1}^{2}-y_{2}^{2}+y_{1}^{2} y_{2}^{2}\right) Y^{2}+\left(2 y_{1} y_{2}-b\right) Y+1=0 .
\end{array}
$$

\subsection{Dihedral group: A linearised YB map}

We replace $(f \widetilde{q}, f p) \rightarrow\left(x_{1}, x_{2}\right)$ in the Darboux matrix (68) to become

$$
M(\mathbf{x} ; k, \lambda)=\left(\begin{array}{cc}
\lambda^{2} f & 0 \\
0 & \lambda^{-2} f
\end{array}\right)+\lambda\left(\begin{array}{cc}
0 & x_{1} \\
x_{2} & 0
\end{array}\right)+f g\left(\begin{array}{cc}
1 & 0 \\
0 & 1
\end{array}\right)+\frac{1}{\lambda}\left(\begin{array}{cc}
0 & x_{2} \\
x_{1} & 0
\end{array}\right) .
$$

Then equations $\Phi_{1}=c_{1}$ and $\Phi_{2}=c_{2}$ imply that the quantities $f$ and $f g$ are given by

$$
\begin{aligned}
f & =\frac{1}{2} \sqrt{1+\left(x_{1}+x_{2}\right)^{2}}+\frac{1}{2} \sqrt{k^{2}+\left(x_{1}-x_{2}\right)^{2}}, \\
f g & =\frac{1}{2} \sqrt{1+\left(x_{1}+x_{2}\right)^{2}}-\frac{1}{2} \sqrt{k^{2}+\left(x_{1}-x_{2}\right)^{2}},
\end{aligned}
$$

where $\left(c_{1}, c_{2}\right) \rightarrow\left(\frac{1-k^{2}}{4}, \frac{1+k^{2}}{2}\right)$.

The linear approximation to the YB map is given by

$$
\left(\begin{array}{l}
x_{1} \\
x_{2} \\
y_{1} \\
y_{2}
\end{array}\right) \stackrel{U_{0}}{\longrightarrow}\left(\begin{array}{l}
u_{1} \\
u_{2} \\
v_{1} \\
v_{2}
\end{array}\right)=\left(\begin{array}{cc|cc}
\frac{(a-1)(a-b)}{(a+1)(a+b)} & \frac{a-b}{a+b} & \frac{2 a}{a+b} & \frac{(a+1)(b-a)}{(b+1)(a+b)} \\
0 & 0 & 0 & \frac{a+1}{b+1} \\
\hline \frac{b+1}{a+1} & 0 & 0 & 0 \\
\frac{(a-b)(b+1)}{(a+1)(a+b)} & \frac{2 b}{a+b} & \frac{b-a}{a+b} & \frac{(b-1)(b-a)}{(b+1)(a+b)}
\end{array}\right)\left(\begin{array}{l}
x_{1} \\
x_{2} \\
y_{1} \\
y_{2}
\end{array}\right)
$$

which is a linear parametric YB map and it is not involutive. 


\section{$52 N \times 2 N$-dimensional YB maps}

In this section we consider the vector generalisations of the YB maps (28) and (61).We replace the variables, $x_{1}$ and $x_{2}$, in the Lax matrices with $N$-vectors $\mathbf{w}_{1}$ and $\mathbf{w}_{2}^{T}$ to obtain $2 N \times 2 N$ YB maps. In what follows we use the following notation for a $n$-vector $\mathbf{w}=$ $\left(w_{1}, \ldots, w_{n}\right)$

$$
\mathbf{w}=\left(\mathbf{w}_{1}, \mathbf{w}_{2}\right), \quad \text { where } \quad \mathbf{w}_{1}=\left(w_{1}, \ldots, w_{N}\right), \quad \mathbf{w}_{2}=\left(w_{N+1}, \ldots, w_{2 N}\right)
$$

and also

$$
\left\langle u_{i}\left|:=\mathbf{u}_{i}, \quad\right| w_{i}\right\rangle:=\mathbf{w}_{i}^{T} \quad \text { and their dot product with } \quad\left\langle u_{i}, w_{i}\right\rangle .
$$

\subsection{NLS equation}

Replacing the variables in (26) with $N$-vectors, namely

$$
M(\mathbf{w} ; a, \lambda)=\left(\begin{array}{cc}
\lambda+a+\left\langle w_{1}, w_{2}\right\rangle & \left\langle w_{1}\right| \\
\left|w_{2}\right\rangle & I
\end{array}\right),
$$

we obtain a unique solution of the Lax-Equation given by the following $2 N \times 2 N$ map

$$
\left\{\begin{array}{l}
\left\langle u_{1}\right|=\left\langle y_{1}\right|+f(z ; a, b)\left\langle x_{1}\right|, \\
\left\langle u_{2}\right|=\left\langle y_{2}\right|
\end{array}\right.
$$

and

$$
\left\{\begin{array}{l}
\left\langle v_{1}\right|=\left\langle x_{1}\right|, \\
\left\langle v_{2}\right|=\left\langle x_{2}+f(z ; b, a)\left\langle y_{2}\right|,\right.
\end{array}\right.
$$

where $f$ is given by

$$
f(z ; b, a)=\frac{b-a}{1+z}, \quad z:=\left\langle x_{1}, y_{2}\right\rangle .
$$

The above is a non-involutive parametric $2 N \times 2 N$ YB map with strong Lax matrix given by (92). As a YB map it appears in [31, but it is originally introduced by Adler [2]. Moreover, one can construct the above $2 N \times 2 N$ map for the $N \times N$ Darboux matrix (92) by taking the limit of the solution of the refactorisation problem in [20].

Two invariants of this map are given by

$$
\begin{array}{r}
I_{1}(\mathbf{x}, \mathbf{y} ; a, b)=\left\langle x_{1}, x_{2}\right\rangle+\left\langle y_{1}, y_{2}\right\rangle, \\
I_{2}(\mathbf{x}, \mathbf{y} ; a, b)=b\left\langle x_{1}, x_{2}\right\rangle+a\left\langle y_{1}, y_{2}\right\rangle+\left\langle x_{1}, y_{2}\right\rangle+\left\langle x_{2}, y_{1}\right\rangle+\left\langle x_{1}, x_{2}\right\rangle\left\langle y_{1}, y_{2}\right\rangle .
\end{array}
$$

These are the invariants which are obtained from the trace of $M(\mathbf{y} ; b, \lambda) M(\mathbf{x} ; a, \lambda)$ and they are not enough to prove Liouville integrability. 


\section{$5.2 \quad \mathbb{Z}_{2}$ reduction}

In the case of $Z_{2}$ we consider, instead of (60), the following matrix

$$
M(\mathbf{w} ; a, \lambda)=\left(\begin{array}{cc}
\lambda^{2}\left(a+\left\langle w_{1}, w_{2}\right\rangle\right) & \lambda\left\langle w_{1}\right| \\
\lambda\left|w_{2}\right\rangle & I
\end{array}\right)
$$

we obtain a unique solution for the Lax-Equation given by the following $2 N \times 2 N$ map

$$
\left\{\begin{array}{l}
\left\langle u_{1}\right|=\left\langle y_{1}\right|+f(z ; a, b)\left\langle x_{1}\right|, \\
\left\langle u_{2}\right|=g(z ; a, b)\left\langle y_{2}\right|
\end{array}\right.
$$

and

$$
\left\{\begin{array}{l}
\left\langle v_{1}\right|=g(z ; b, a)\left\langle x_{1}\right|, \\
\left\langle v_{2}\right|=\left\langle x_{2}\right|+f(z ; b, a)\left\langle y_{2}\right|,
\end{array}\right.
$$

where $f$ and $g$ are given by

$$
f(z ; a, b)=\frac{a-b}{a-z}, \quad g(z ; a, b)=\frac{a-z}{b-z}, \quad z:=\left\langle x_{1}, y_{2}\right\rangle .
$$

The above map is a non-involutive parametric $2 N \times 2 N$ YB map with strong Lax matrix given by (98).

The invariants of the above map, coming from the trace of $M(\mathbf{y} ; b, \lambda) M(\mathbf{x} ; a, \lambda)$, are given by

$$
\begin{aligned}
& I_{1}(\mathbf{x}, \mathbf{y} ; a, b)=\left\langle x_{1}+y_{1}, x_{2}+y_{2}\right\rangle \\
& I_{2}(\mathbf{x}, \mathbf{y} ; a, b)=b\left\langle x_{1}, x_{2}\right\rangle+a\left\langle y_{1}, y_{2}\right\rangle+\left\langle x_{1}, x_{2}\right\rangle\left\langle y_{1}, y_{2}\right\rangle
\end{aligned}
$$

In fact, both vectors of the inner product in $I_{1}$ are invariants. However, as in the vector generalisation of the Adler-Yamilov map, the invariants are not enough to claim Liouville integrability.

The Liouville integrability of the vector generalisations of the YB maps we consider in this section is an open problem.

\section{Acknowledgements}

We would like to thank A. Veselov, T. Kouloukas, V. Papageorgiou, A. Dzhamay and G. Grahovski for the discussions and their comments, P. Xenitidis for helping to improve the text and special thanks to D. Tsoubelis for making available his computing facilitites. A.V.M. would like to acknowledge support from EPSRC (EP/I038675/1). S.K.R. would like to acknowledge William Right Smith scholarship and John E. Crowther scholarship. 


\section{References}

\section{References}

[1] Adler V 1993 Recuttings of polygons Funktsional. Anal. i Prilozhen. 27 79-82.

[2] AdLeR V 1994 Nonlinear superposition principle for the Jordan NLS equation Phys. Lett. A $19053-58$.

[3] Adler V, Bobenko A, And Suris Y 2003 Classification of integrable equations on quad-graphs. The consistency approach Comm. Math. Phys. 233 513-543.

[4] Adler V, Bobenko A, And Suris Y 2004 Geometry of Yang-Baxter maps: pencils of conics and quadrirational mappings Comm. Anal. Geom. 12 967-1007.

[5] Adler V And Yamilov R 1994 Explicit auto-transformations of integrable chains $J$. Phys. A 27 477-492.

[6] Baxter R 1972 Partition function of the eight-vertex lattice model Ann. Physics 70 $193-228$.

[7] Bobenko A and Suris Y. 2002 Integrable systems on quad-graphs Int. Math. Res. $11573-611$.

[8] BuRY R 2010 Automorphic Lie algebras, corresponding integrable systems and their soliton solutions Ph.D. thesis, Un. of Leeds.

[9] Bury R And Mikhailov A 2012 Automorphic Lie algebras and corresponding integrable systems. I. to be submitted

[10] Cai H, Liu F, And Huang N 2003 Hamiltonian formalism of the derivative nonlinear Schrödinger equation Commun. Theor. Phys. 39 181-188.

[11] CieŚLiński J 1995 An algebraic method to construct the Darboux matrix J. Math. Phys. 36 5670-5706.

[12] Drinfel'D V 1992 On some unsolved problems in quantum group theory Lecture Notes in Math. 1510 1-8.

[13] Fordy A 2012 Integrable Poisson maps from cluster exchange relations Lecture notes

- SIDE summer school, Ningbo.

[14] Fordy A And Kassotakis A 2013 Integrable Maps which Preserve Functions with Symmetries J. Phys. A 46205201.

[15] Kassotakis P and Nieszporski M 2012 On non-multiaffine consistent-around-thecube lattice equations Phys. Lett. A 376 3135-3140.

[16] Kaup D And Newell A 1978 An exact solution for a derivative nonlinear Schrödinger equation J. Mathematical Phys. 19 798-801. 
[17] Konstantinou-Rizos S, Mikhailov A, and Xenitidis P 2012 Reduction groups and integrable difference systems of NLS type To be submitted.

[18] Kouloukas T 2010 Yang-Baxter maps, poisson structure and integrability Ph.D thesis, Un. of Patras, Greece.

[19] Kouloukas T and Papageorgiou V 2009 Yang-Baxter maps with first-degreepolynomial $2 \times 2$ Lax matrices J. Phys. A 42404012 .

[20] Kouloukas T and Papageorgiou V 2011 Poisson yang-baxter maps with binomial lax matrices J. Math. Phys. 52073502.

[21] Lombardo S 2004 Reductions of integrable equations and automorphic Lie algebras Ph.D. thesis, Un. of Leeds.

[22] Lombardo S And Mikhailov A 2005 Reduction groups and automorphic Lie algebras Comm. Math. Phys. 258 179-202.

[23] Matveev V and Salle M 1991 Darboux transformations and solitons Integrable systems in statistical mechanics, Springer series in nonlinear dynamics.

[24] Mikha ĬLOV A 1981 The reduction problem and the inverse scattering method Physica D 3 73-117.

[25] Mikha Ǐlov A, Shabat A, and Yamilov R 1988 Extension of the module of invertible transformations. Classification of integrable systems Comm. Math. Phys. 115 1-19.

[26] Nijhoff F and Capel H 1995 The discrete Korteweg-de Vries equation Acta Appl. Math. 39 133-158.

[27] NiJhoff F 2002 Lax pair for the Adler (lattice Krichever-Novikov) system Phys. Lett. A $29749-58$.

[28] Nijhoff F 2010 Discrete systems and integrability Academic lecture notes.

[29] Nijhoff F And Walker A 2001 The discrete and continuous Painlevé VI hierarchy and the Garnier systems Glasg. Math. J. 43A 109-123.

[30] Papageorgiou V, Nijhoff F, and Capel H 1990 Integrable mappings and nonlinear integrable lattice equations Phys. Lett. A 147 106-114.

[31] Papageorgiou V and Tongas A 2007 Yang-Baxter maps and multi-field integrable lattice equations J. Phys. A 40 12677-12690.

[32] Papageorgiou V, Tongas A, and Veselov A 2006 Yang-Baxter maps and symmetries of integrable equations on quad-graphs J. Math. Phys. 47 083502, 16.

[33] Rogers C And Schief W 2002 Bäcklund and Darboux transformations. Geometry and modern applications in soliton theory, Cambridge texts in applied mathematics. 
[34] SuRIS Y And Veselov A 2003 Lax matrices for Yang-Baxter maps J. Nonlinear Math. Phys. 10 223-230.

[35] Veselov A 1991 Integrable maps Russ. Math. Surveys 46 1-51.

[36] Veselov A 2003 Yang-Baxter maps and integrable dynamics Phys. Lett. A $314214-$ 221.

[37] Veselov A 2007 Yang-Baxter maps: dynamical point of view J. Math. Soc. Japan 17 $145-167$.

[38] YANG C 1967 Some exact results for the many-body problem in one dimension with repulsive delta-function interaction Phys. Rev. Lett. 19 1312-1315.

[39] Zaharov V AND ŠABAT A 1979 Integration of the nonlinear equations of mathematical physics by the method of the inverse scattering problem. II Funktsional. Anal. $i$ Prilozhen. 13 13-22. 